\title{
Using bluetooth to implement a pervasive indoor positioning system with minimal requirements at the application level
}

\author{
Mario Muñoz-Organero*, Pedro J. Muñoz-Merino and Carlos Delgado Kloos \\ University Carlos III of Madrid, Madrid, Spain
}

\begin{abstract}
Different systems have been proposed to estimate the position of a mobile device using Bluetooth based on metrics such as the Radio Signal Strength Indicator (RSSI), the received Bit Error Rate (BER) or the Cellular Signal Quality (CSQ). These systems try to improve the estimation accuracy of the basic and straightforward triangulation method among discovered BT reference base stations at the cost of requiring that the positioning application has access to low level hardware related data (provided by the Host Controller Interface) and obtaining information which is in many cases hardware, and therefore device, dependent. In this paper we design, simulate, implement and validate a Bluetooth positioning system that only requires the ability to handle SDP service records at the application level, achieving mean errors around 1 to 3 meters, improving the basic triangulation method among discovered BT reference base stations.
\end{abstract}

Keywords: Indoor positioning systems, pervasive computing, mobile computing, mobile systems, mobile applications

\section{Introduction}

Location Based Services can be considered as one of the most rapidly expanding fields of the mobile communications sector [26]. The limits of global, satellite based, positioning systems such as GPS to provide location information in indoors environments have fostered the use of different wireless technologies to locate mobile devices in such environments. Some of the former systems such as Active Bat [1], Cricket [2] and DOLPHIN [3] were based on ultrasonic signals, providing accurate estimations with mean errors of a few centimetres but requiring significant manual configuration and covering small areas. WLAN based positioning systems such as RADAR [4], WiPS [5-7] have been defined to provide location information in wider areas with positioning accuracy of some meters. LANDMARC [8] and SpotON [9] make use of RFID for cost-effective indoor locating of objects in reduced areas such as rooms with medium accuracy depending on the number of elements. Bluetooth has also been used for the definition of positioning systems such as [10-19] covering small to medium size areas with a few meters accuracy. There are also systems that try to improve the accuracy by combining several technologies. DOLPHIN [3] combines ultrasounds and RF signals. The system in [20] combines Bluetooth and WLAN to improve accuracy in indoor positioning systems. Moreover, there are systems that try to provide a combined indoor-outdoor solution for positioning devices [21-23].

There are however some practical limitations when trying to deploy the previous systems for indoor positioning in pervasive environments containing user devices including both mobile phones and PDAs.

\footnotetext{
${ }^{*}$ Corresponding author: Mario Muñoz-Organero, University Carlos III of Madrid, Av. Universidad 30, E-29811 Leganés, Madrid, Spain. Tel.: +34 916248801; Fax: +34 916248749; E-mail: munozm@it.uc3m.es.
} 
WLAN technologies, although extended in PDAs and smart phones, are not pervasive enough in nonsmart mobile phones. Ultrasonic signal receivers are not a common feature in mobile phones or PDAs and RFID is currently been explored only in a limited number of devices with the implementation of NFC [24]. Bluetooth is a more pervasive technology among mobile phones and PDAs [28]. However previously mentioned positioning systems based on Bluetooth [10-19] tend to use metrics such as the Radio Signal Strength Indicator (RSSI), the received Bit Error Rate (BER) or the Cellular Signal Quality (CSQ) to try to improve the estimation accuracy of the basic and straightforward triangulation method among discovered BT reference base stations, requiring that the positioning application has access to low level hardware related data (provided by the Host Controller Interface) and obtaining information which is in many cases hardware, and therefore device, dependent. Trying to optimize the network performance by modifying the standard protocol parameters such as in [27] restricts the ubiquity of the solution. Forgen et al. [25] analyzed the need for a localization API in mobile devices that should be more transparent and more integrated to applications. We have designed, simulated, implemented and validated a new Bluetooth based positioning system which minimizes the requirements from the underlying hardware interface and obtains at least similar accuracy than previously mentioned systems.

In this paper we will first review former proposals for Bluetooth based indoor positioning systems to later describe and justify our proposal. We will provide simulation as well as real measurements to validate the accuracy of our systems.

\section{Bluetooth-based indoor positioning systems}

Bluetooth defines the HCI (Host Controller Interface) through which some measures of the received power level of the hardware interface are made available to positioning applications. Feldmann et al. [12] propose a Bluetooth positioning system based on an approximation of the relation between the Radio Signal Strength Indicator (RSSI) and the associated distance between sender and receiver. The system obtains a precision of 2,08 meters and the authors propose that it should be combined with an inertial systems and an adequate Kalman Filter. Zhou and Pollard [18] define a mechanism to improve the use of the Radio Signal Strength Indicator (RSSI) for Bluetooth indoor positioning systems by modifying the standard Bluetooth behaviour by disabling the automatic transmitter power control based on the RSSI measurements. These authors compare theoretical results with real measures showing that there are some factors such as multipath fading that affect the results. Thapa and Case [14] propose the design of a Bluetooth indoor positioning system which combines the Link Quality (LQ), Radio Signal Strength Indicator (RSSI) and Transmit Power Level (TPL) obtained from the Host Controller Interface (HCI) to improve the accuracy of former RSSI based systems. The paper does not present evaluation results however. A different system [15] that combines the measures of RSSI, LQ and the Cellular Signal Quality (CSQ) is able to obtain accuracies of less than 1 meter but only for a reduced area of a few square meters and requiring the realization of tedious system calibration measurements. Son and Orten [19] propose a different approach for Bluetooth positioning based on the time offset acquisition of signals propagating over radio channels which requires the modification of the standard Bluetooth technology. These authors present results of up to 1,5 meters of accuracy.

There are also other related studies about how to improve the accuracy provided by Bluetooth positioning systems. Genco et al [17] propose a mechanism to determine the optimal locations for deploying Bluetooth base stations taking into account the restrictions and constrains required by the physical setting. This proposal can minimize the number of Bluetooth base stations required for obtaining a given accuracy. Bruno and Delmastro [16] propose an infrastructure orchestrated system. Kelly et al. [15] also 
propose a system orchestrated by infrastructure nodes in which the number of these infrastructure nodes is reduced to 1 node per room.

All of these systems present some limitations when trying to deploy them on mobile devices such as commercially available mobile phones in order to implement a pervasive Bluetooth based positioning system. On the one hand, modifications to the Bluetooth specifications should be avoided. On the other hand, systems requiring calibration for particular devices before their use should also be discouraged. Furthermore, the availability of HCI information to user applications is not always defined for certain programming environments such as the micro-edition version of Java (J2ME). We present a new Bluetooth positioning system that deals with these limits and at the same time provides similar accuracy by improving the basic and straightforward triangulation method among discovered BT reference base stations.

\section{A bluetooth indoor positioning system with minimal requirements at the application level}

A pervasive feature of Application Programming Interfaces (APIs) for developing Bluetooth based applications is the ability to discover nearby Bluetooth devices and the services they offer. Our system is based on the use of Service Records made available by the Bluetooth Service Discovery Protocol (SDP) to provide the location information of Bluetooth base stations. A mobile Bluetooth device can discover nearby base stations and obtain their location by reading the information provided in these Service Records. We describe, in this section, 3 different mechanisms to combine the location information of the detected Bluetooth base stations in order to estimate the position of a mobile device that will be evaluated and tested in the next sections in order to validate if any of them can be used to define a pervasive Bluetooth positioning system. The first one represents the basic and straightforward triangulation method among discovered BT reference base stations which will be used as a reference to compare the accuracy improvements by the other 2 mechanisms.

A simple way to combine the location information of detected base stations was proposed in [8] for a different system based on RFID active tags and has been used in simple BT based positioning applications. If the location of each base station is $\left(x_{i}, y_{i}, z_{i}\right)$ the location of the mobile device can be estimated as:

$$
(x, y)=\sum_{i=1}^{k} \omega_{i}\left(x_{i}, y_{i}, z_{i}\right),
$$

where $\omega_{i}$ is the weighting factor to the ith neighboring base station. The basic triangulation method among discovered BT reference base stations defines a system in which all $\omega_{i}$ are $1 / k$ being $k$ the number of detected Bluetooth base stations.

Our first proposal for improving the accuracy of the system while restricting the required data to the location of detected BT base stations tries to improve the results by using weights that compensate a non homogeneous density or special distribution of base stations. We define the weights in Eq. (1) to be:

$$
\begin{aligned}
& \omega_{i}^{\prime}=\sum_{j=1}^{k} \sqrt{\left(x_{i}-x_{j}\right)^{2}+\left(y_{i}-y_{j}\right)^{2}+\left(z_{i}-z_{j}\right)^{2}} \\
& \omega_{i}=\frac{\omega_{i}^{\prime}}{\sum_{j=1}^{k} \omega_{j}^{\prime}}
\end{aligned}
$$


which represents the normalized cumulative Euclidean distance between the ith base station and the rest of the base stations. The idea is that the bigger the distance the more isolated a base station is and therefore the spatial density in the area of that base station is smaller and therefore this should be compensated with a bigger weight.

Our second proposal is based on selective triangulation. If we select 3 of the detected base stations we can try to estimate position of the mobile device by taking into account the overlapping zone of the spheres centred in each of these base stations and with a radius dependent of the RF coverage (typically 10 meters). The overlapping zone is big if the base stations are close to each other but its size is reduced if the relative distance between the selected base stations increases. Our proposal selects the 3 base stations that maximize the formula:

$$
\begin{aligned}
d_{i, j, k} & =\sqrt{\left(x_{i}-x_{j}\right)^{2}+\left(y_{i}-y_{j}\right)^{2}+\left(z_{i}-z_{j}\right)^{2}} \\
& +\sqrt{\left(x_{i}-x_{k}\right)^{2}+\left(y_{i}-y_{k}\right)^{2}+\left(z_{i}-z_{k}\right)^{2}} \\
& +\sqrt{\left(x_{k}-x_{j}\right)^{2}+\left(y_{k}-y_{j}\right)^{2}+\left(z_{k}-z_{j}\right)^{2}},
\end{aligned}
$$

and estimates the position of the mobile device as the point which is equidistant to these three base stations (the central point of the overlapping zone).

To estimate the accuracy that these alternatives can provide depending on the number and location of the Bluetooth base stations we have developed a simulator that receives the number of base stations, distributes them randomly and performs the calculation of the estimated position of a mobile device by each of these options. The results are presented in the next section.

\section{Simulated results}

In order to evaluate the mean accuracy of the methods for estimating the position of mobile devices described in the previous section we have developed a simulator based on a generic spherical RF coverage model. The input parameters are the coverage range, the number of Bluetooth base stations, the calculation method and the number of iterations. For each iteration, the base stations are randomly distributed inside the coverage range of the user situated in a predefined location in the centre of the simulated area. The mean error taking into account the errors in each iteration is then calculated and reported as the output of the simulation.

Figure 1 presents the results for the mean error of the estimated distance after 2000 iterations for each of the three methods (the first one, the simple triangulation method, is used for comparison purposes to evaluate the accuracy improvements of methods 2 and 3) when the number of Bluetooth base stations in the coverage range of the mobile device varies from 3 to 15 . The $x$ axis represents the number of base stations. The $y$ axis represents de mean location error in meters. The accuracy of the three methods is similar in the presence of only 3 base stations. As the number of Bluetooth base stations increases the accuracy of method 3 gets better than method 2 which gives better results than method 1. For 15 base stations, for example, the mean error for method 3 is 1,29 meters while method 2 provides a mean error of 1,58 meters and method 1 of 1,78 meters. A possible argumentation that explains why method 2 performs better than method 1 is because the distribution of the base stations is random and therefore there are many cases in which the density of base stations is not homogeneous. A possible of argumentation that explains why method 3 performs better than method 2 and method 1 when the number of base stations 


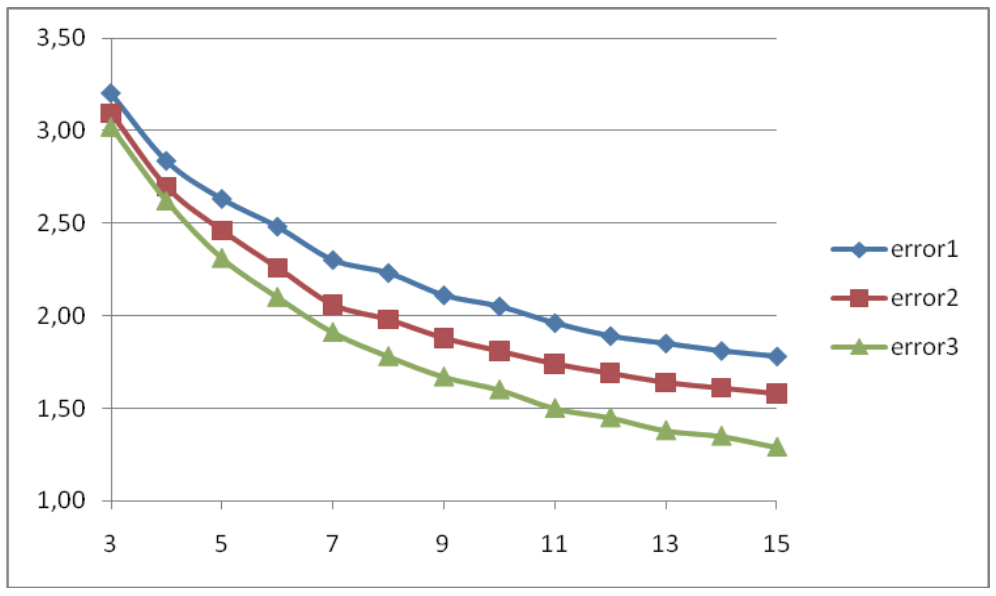

Fig. 1. Simulated accuracy for the 3 proposed methods.

increases is that the probability to find base stations near the border of the coverage area of the mobile device also increases and the resulting errors are therefore minimized.

In real scenarios is practical and desirable to deploy the minimum number of Bluetooth base stations as possible while providing acceptable results. In order to minimize the required number of base stations, the user mobile devices acting as clients in the system to be located can also be activated to provide location information. In environments such as museums, fairs or shopping centres the mean density of users could be significant for our positioning system. We have extended our simulator to incorporate mobile devices as location providers. In order to do this, a random location error which depends on the previously simulated errors as a function of the number of stationary Bluetooth base stations is introduced to each mobile device. Therefore, each mobile device acts as a Bluetooth base station that provides information about a location which is the correct location of the mobile device plus a random error. The location of each mobile device is therefore a random variable with the distribution calculated in the previous simulation. A limit scenario in which there are only 3 stationary base stations in the coverage range of the mobile device to be located has been simulated. The number of mobile devices providing location information has been varied from 0 to 12 . This simulation results are captured in Fig. 2. The $x$ axis represents the number of base stations ( 3 of them are stationary). The $y$ axis represents de mean location error in meters. In the case of 0 mobile users providing location information the results are similar to those in Fig. 1. Method 2 continues to provide better results than method 1. However, method 3 only provides slightly better results than method 2 for a limited number of mobile devices acting as location providers. If the number of these devices increases method 2 outperforms method 3 . This is due to the fact that method 3 only takes into account the 3 stations with bigger relative distances while method 2 takes all the stations into consideration and, therefore, random errors are better compensated.

Figure 3 captures a different scenario in which we always have 3 mobile stations and a variable number of stationary based stations. The $x$ axis represents the number of base stations ( 3 of them are mobile). The $y$ axis represents de mean location error in meters. The accuracy of method 3 is improved as the number of stationary base stations is increased since the probability that the 3 selected base stations for the calculations are error free also increases.

The simulated results have shown that accep Table l ocation errors of less than 2 meters can be obtained with a limited number of 8 base stations ( 3 of them can be mobile devices) per each 314 square meters area (assuming a maximum coverage range of 10 meters). 


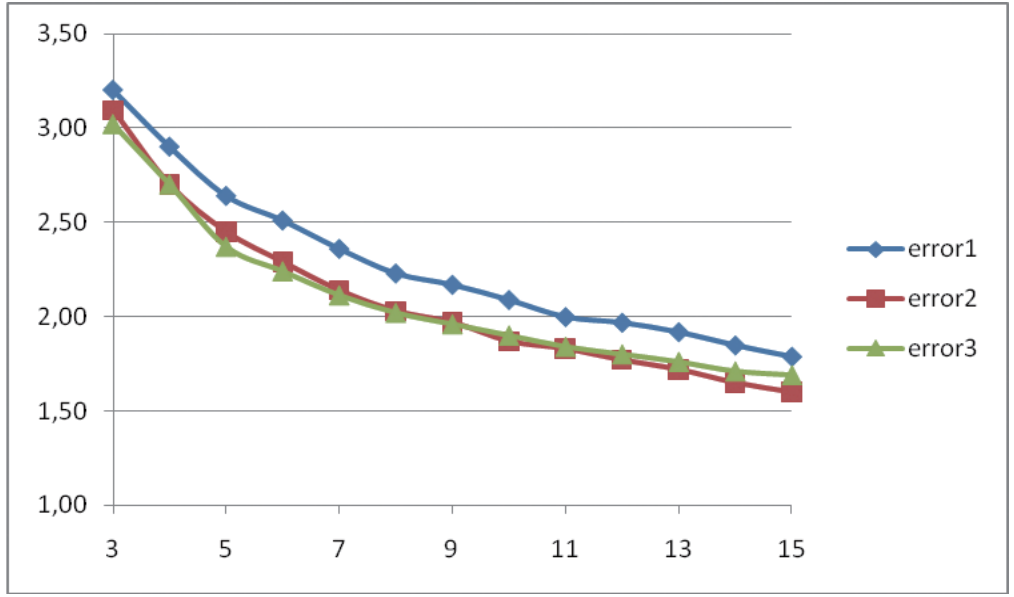

Fig. 2. Simulated accuracy for the 3 proposed methods in the presence of a variable number of mobile devices providing location information.

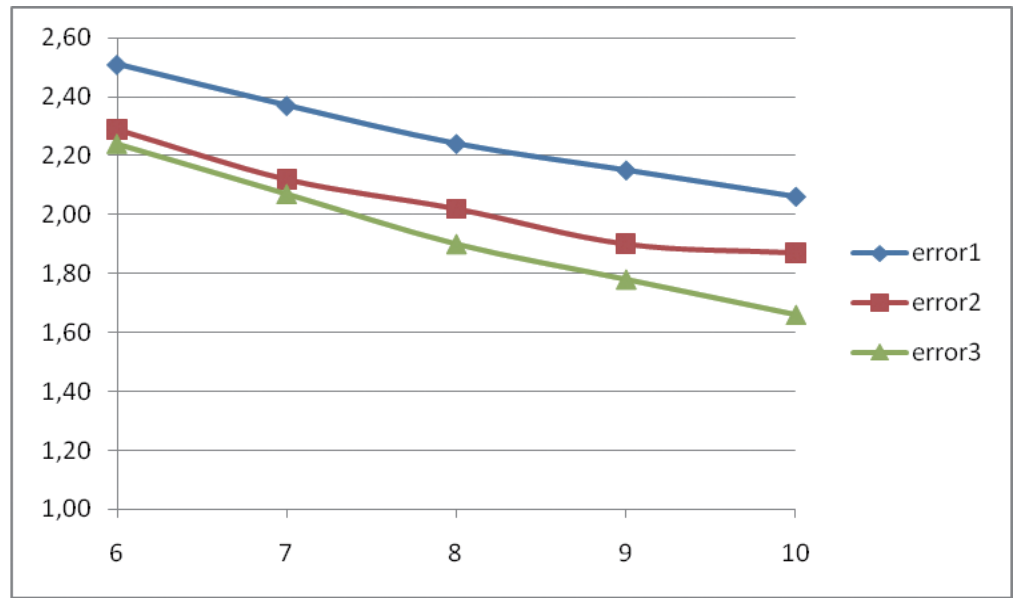

Fig. 3. Simulated accuracy for the 3 proposed methods in the presence of 3 mobile devices providing location information.

The free space propagation model implemented in the simulator may be not accurate for real indoor scenarios. We have therefore implemented our system in several scenarios with real devices to compare the results with the simulations.

\section{Validation of results in real scenarios}

In order to validate the simulated results, the applications needed to deploy our Bluetooth based positioning system were implemented in Java, both in J2ME and J2SE. J2ME provides a native API (JSR82) for interacting with the Bluetooth stack. An implementation of a similar API (Bluecove) was used for J2SE. Figure 4 shows some example screens of the base station application and the client application in J2ME on one of the mobile phones used.

The first scenario is a 30 meters corridor with only 3 stationary base stations (running on Nokia 6131 NFC mobile devices) separated 10 meters and installed on the same wall, 2 metres from the ground. A 
Table 1

Points at which the position of the mobile device was estimated

\begin{tabular}{lcc}
\hline Locations & $\mathrm{X}(\mathrm{m})$ & $\mathrm{Y}(\mathrm{m})$ \\
\hline 1 & 5 & 4 \\
2 & 1 & 1 \\
3 & 1 & 4 \\
4 & 5 & 5 \\
5 & 9 & 1 \\
6 & 9 & 5 \\
\hline
\end{tabular}
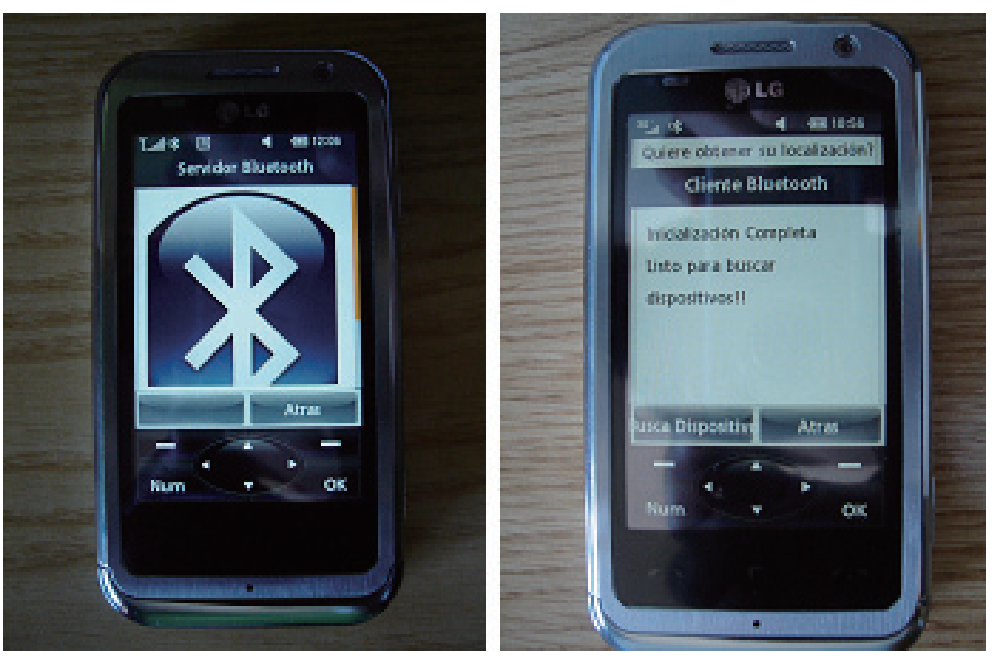

Fig. 4. Implemented applications in J2ME (base station application on the left and client application on the right).

single mobile device was used to get the location every 2 meters. Only method 1 was used (since the number of Bluetooth base stations was very small). The mean error obtained was 2,27 meters.

A second scenario is captured in Fig. 5. The scenario represents the floor of a building with 5 stationary Bluetooth base stations (implemented with heterogeneous devices including a Dell laptop, two Nokia 6131 and two Nokia 6212 mobile phones) and a mobile user device whose position is going to be estimated. Figure 1 captures the locations of the base stations. The Bluetooth device in the centre of the figure inside a square box was a mobile device not considered for this second scenario. Table 1 captures the points in which the position of the "moving" mobile device was obtained. In order to maximize the effects of the obstacles in the measures taken (as a worst case scenario), all the doors were closed. The mean error estimated by method 1 has been 1,48 meters. Method 2 provided a mean error of 1,41 meters and method 3 estimated the positions with a mean error of 1,39 meters.

A third scenario introduced the mobile device inside the square box in Fig. 1 as a mobile base station. The position estimation points were the same as captured in Table 1. In this scenario, the mean error estimated by method 1 improved to 1,37 meters while method 3 estimations provided a slightly bigger mean error of 1,45 meters. This result can be justified based on the simulations carried out in the previous section. Method 3 tends to degrade its estimations when the relative number of mobile base stations compared to stationary base stations increases. In this scenario, there are some points in Table 1 in which there where only Bluetooth coverage of 2 stationary base stations and the newly introduced mobile base station. 


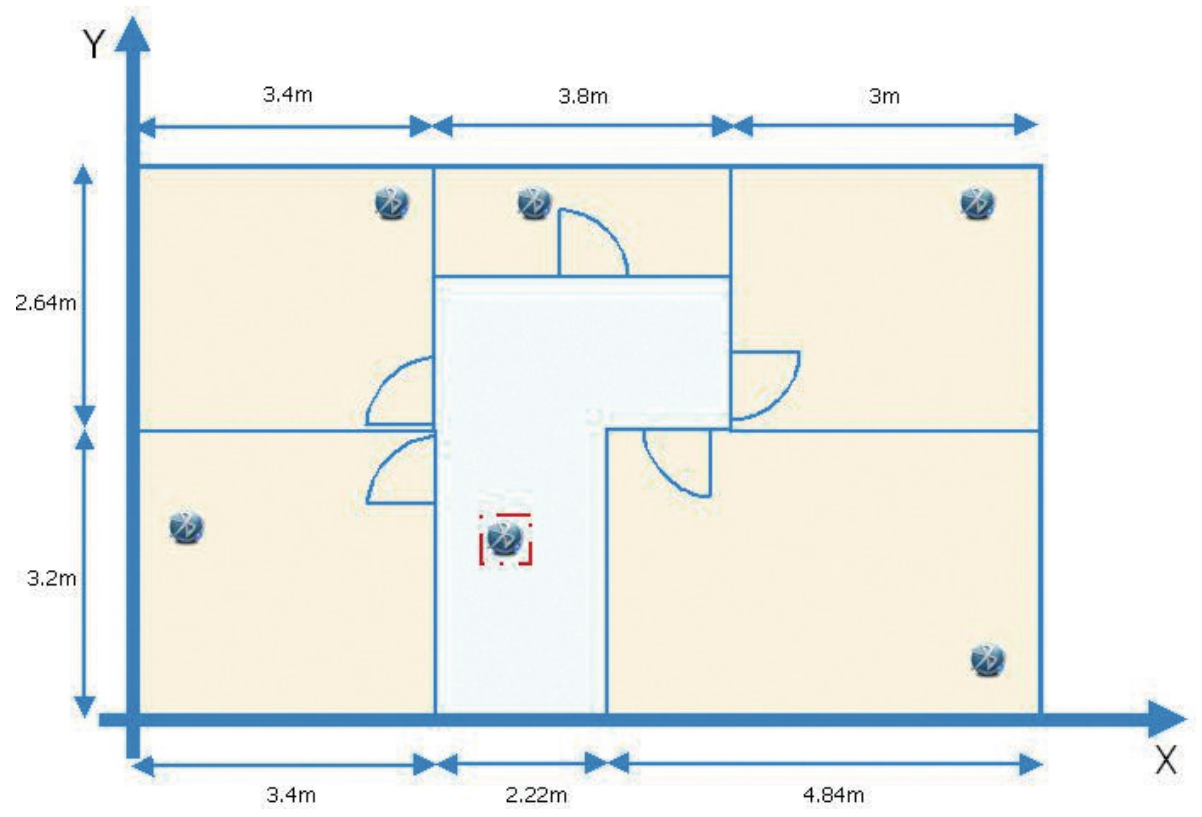

Fig. 5. Implemented scenario.

\subsection{Conclusions}

A new Bluetooth based positioning system for indoor environments has been presented in this paper. The system tries to minimize the requirements that the positioning application needs from the underlying Bluetooth APIs. The system is based on the use of SDP service records to send the coordinates of each base station. Previous Bluetooth positioning systems are based on metrics such as the Radio Signal Strength Indicator (RSSI), the received Bit Error Rate (BER) or the Cellular Signal Quality (CSQ) which require the positioning application to have access to low level hardware related information (provided by the Host Controller Interface). The mean accuracy obtained by the use of our new Bluetooth based positioning system for indoor environments is similar to previous systems (in the range of 1 to 3 meters depending on the number and distribution of Bluetooth base stations).

We have proposed two methods for estimating the position of a Bluetooth mobile device based on the position of discovered Bluetooth base stations which improve the simple calculation of the average coordinates of the positions of the detected base stations. The first proposed method improves the accuracy by introducing compensating weights for the non-regular density distribution of base stations. The weights take into account the cumulative distances among the discovered base stations. The second proposed method bases the position estimations on triangulation. Among the discovered base stations the method estimates the 3 which are further from the user in order to reduce the overlapping zone of the intersecting spheres of Bluetooth radio coverage.

The paper presents the results both of several simulations and real cases. The simulations did not include the presence of obstacles but provided a mechanism to compare the 2 methods proposed with the simple calculation of the average coordinates of the positions of the detected base stations. Three real cases were used to validate the results in the presence of obstacles in indoor scenarios.

The simulations showed an increase in accuracy of the position estimating methods as the number of Bluetooth base stations also increased. In order to minimize the requirements from the infrastructure 
deployment, the paper has also proposed, analysed, simulated and validated the inclusion of mobile Bluetooth devices as base stations. The position of these mobile devices can be simulated as a random variable whose distribution can be approximated by the simulations with stationary base stations. Both the simulations and the implemented real scenarios showed a better relative performance of the proposed methods compared with the basic triangulation method among discovered BT reference base stations.

\section{Acknowledgments}

The research leading to these results has received funding by the ARTEMISA project TIN200914378-C02-02 within the Spanish "Plan Nacional de I+D+I", and the Madrid regional community projects S2009/TIC-1650 and CCG10-UC3M/TIC-4992.

\section{References}

[1] A. Harter, A. Hopper, P. Steggles, A. Ward and P. Webster, The Anatomy of a Context-Aware Application, 5th Annual ACM/IEEE Int. Conference on Mobile Computing and Networking, Seattle USA, Aug 1999, pp. 59-68.

[2] N.B. Priyantha, A. Chakraborty and H. Balakrisnan, The Cricket Location-Support System, 6th Int. Conference on Mobile Computing and Networking, Aug 2000, pp. 32-43.

[3] Y. Fukuju, M. Minami, H. Morikawa and T. Aoyama, DOLPHIN: An Autonomous Indoor Positioning System in Ubiquitous Computing Environment, In Proceedings of the IEEE Workshop on Software Technologies For Future Embedded Systems. WSTFES. IEEE Computer Society, Washington, DC, 53, May 2003.

[4] P. Bahl and V.N. Padmanabhan, RADAR: An In-Building RF-based User Location and Tracking System, INFOCOM 2000. Nineteenth Annual Joint Conference of the IEEE Computer and Communications Societies, Vol.2 IEE Proceedings, pp. 775-784.

[5] T. Kitasuka, K. Hisazumi and T. Nakanishi, WiPS: Location and Motion Sensing Technique of IEEE 802.11 Devices, Third International Conference on Information Technology and Applications, 2005, ICITA, pp. 346-349.

[6] Y. Wang, X. Jia and H. Lee, An indoors wireless positioning system based on wireless local area network infrastructure, in Proceedings of the 6th International Symposium on Satellite Navigation Technology Including Mobile Positioning and Location Services, 2003.

[7] J. Yang and Y. Chen, Indoor localization using improved RSS-based lateration methods, In Proceedings of the 28th IEEE Conference on Global Telecommunications (Honolulu, Hawaii, USA). M. Ulema, Ed. IEEE Press, Piscataway, NJ, Nov-Dec 2009, pp. 4506-4511.

[8] L.M. Ni, Y. Liu, Y.C. Lau and A.P. Patil, LANDMARC: Indoor Location Sensing Using Active RFID, Wireless Networks, ed., Springer Netherlands, 2004, pp. 701-710.

[9] J. Hightower, G. Borriello and R. Want, SpotON: An indoor 3D Location Sensing Technology Based on RF Signal Strength, UW CSE, Technical Report \#2000-02-02.

[10] J. Yipin Ye, Atlantis: Location Based Services with Bluetooth, Master's Thesis, Brown University, 2005.

[11] F. Forno, G. Malnati and G. Portelli, Design and Implementation of a Bluetooth ad hoc Network for Indoor Positioning, IEE Proceedings-Softw 152 (2005), 223-228.

[12] S. Feldmann, K. Kyamakya, A. Zapater and Z. Lue, An Indoor Bluetooth-Based Positioning System: Concept, Implementation and Experimental Evaluation, International Conference on Wireless Networks, Las Vegas, 2003.

[13] U. Bandara, M. Hasegawa, M. Inoue and H. Morikawa, Design and Implementation of a Bluetooth Signal Strength Based Location Sensing System, Radio and Wireless Conference, IEEE, 2004, pp. 319-322.

[14] K. Thapa and S. Case, An indoor positioning service for Bluetooth Ad Hoc networks, in: MICS 2003, Duluth, MN, USA.

[15] D. Kelly, S. McLoone and T. Dishongh, A Bluetooth-Based Minimum Infrastructure Home Localisation System, n Proceedings of 5th IEEE International Symposium on Wireless Communication Systems (ISWCS 2008), pp. 638-642.

[16] R. Bruno and F. Delmastro, Design and analysis of a Bluetooth-based indoor localization system, PersonalWireless Communications (2003), 711-725.

[17] A. Genco, S. Sorce and G. Scelfo, Bluetooth Base Station Minimal Deployment for High Definition Positioning, Proceedings of the The Second Annual International Conference on Mobile and Ubiquitous Systems: Networking and Services, July 17-21, 2005, pp. 454-460. 
[18] S. Zhou and J.K. Pollard, Position measurement using Bluetooth, IEEE Transactions on Consumer Electronics 52(2) (2006), 555-558.

[19] L.T. Son and P. Orten, Enhancing Accuracy Performance of Bluetooth Positioning, in proceedings of the IEEE Wireless Communications and Networking Conference (WCNC 2007), Hong Kong, March 2007.

[20] S. Aparicio, J. Perez, A. Bernardos and J. Casar, A Fusion Method Based on Bluetooth and WLAN Technologies for indoor location, in Proceedings of IEEE Conference on Multisensor Fusion and Integration for Intelligent Systems, 2008, pp. 487-491.

[21] A. Lamarca, Y. Chawathe, S. Consolvo, J. Hightower, I. Smith, J. Scott, T. Sohn, J. Howard, J. Hughes, F. Potter, J. Tabert, P. Powledge, G. Borriello and B. Schilit, Place lab: Device positioning using radio beacons in the wild, in Proceedings of the Third International Conference on Pervasive Computing, May 2005.

[22] L. Pei, R. Chen, Y. Chen, H. Leppakoski and A. Perttula, Indoor/Outdoor Seamless Positioning Technologies Integrated on Smart Phone, Advances in Satellite and Space Communications, International Conference on, 2009 First International Conference on Advances in Satellite and Space Communications, 2009, pp. 141-145.

[23] L. Reyero and G.Y. Delisle, Always best located, a pervasive positioning system. InWireless Pervasive Computing, 2007. ISWPC '07. 2nd International Symposium on, 2007.

[24] T. Wiechert, F. Thiesse, F. Michahelles, P. Schmitt and E. Fleisch, Connecting Mobile Phones to the Internet of Things: A Discussion of Compatibility Issues between EPC and NFC, Americas Conference on Information Systems (AMCIS), 2007, Keystone, Colorado, USA.

[25] A. Fongen, C. Larsen, G. Ghinea, S.J.E. Taylor and T. Serif, Location based mobile computing - A tuplespace perspective, Mobile Information Systems, 2006, pp. 135-149.

[26] I. Priggouris, D. Spanoudakis, M. Spanoudakis and S. Hadjiefthymiades, A generic framework for Location-Based Services (LBS) provisioning, Mobile Information Systems (2006), 111-133.

[27] M. Goyal, W. Xie and H. Hosseini, IEEE 802.15.4 modifications and their impact, Mobile Information Systems, 2011, pp. 69-92

[28] Y. Li, H. Chen, R. Xie and J.Z. Wang, BGN: A novel scatternet formation algorithm for bluetooth-based sensor networks, Mobile Information Systems, 2011, pp. 93-106.

Mario Muñoz-Organero is an associate professor of telematics engineering at Carlos III University of Madrid. His research interests include e-learning, m-learning, open architectures for e-learning systems, open service-creation environments for next-generation networks, advanced mobile communication systems, pervasive computing, and convergent networks. Muñoz-Organero has a PhD in telecommunications engineering from Carlos III University of Madrid. Contact him at munozm@it.uc3m.es.

Pedro J. Muñoz-Merino is a visiting professor of telematics engineering at Carlos III University of Madrid. His primary research interests are in e-learning. Muñoz-Merino has a $\mathrm{PhD}$ in telecommunications engineering from Carlos III University of Madrid, Spain. Contact him at pedmume@it.uc3m.es.

Carlos Delgado Kloos is a professor of telematics engineering at Carlos III University of Madrid, where he was founding director of the Department of Telematics Engineering and is currently vice chancellor, director of two master's programs (one on e-learning), and director of the Nokia Chair. His research interests are in educational technologies. He coordinated the Europeanfunded E-LANE project on e-learning in Latin America and was a member of the .LRN Consortium's board of directors. Delgado Kloos has a PhD in computer science from the Technical University of Munich and a $\mathrm{PhD}$ in telecommunication engineering from the Technical University of Madrid. Contact him at cdk@it.uc3m.es. 

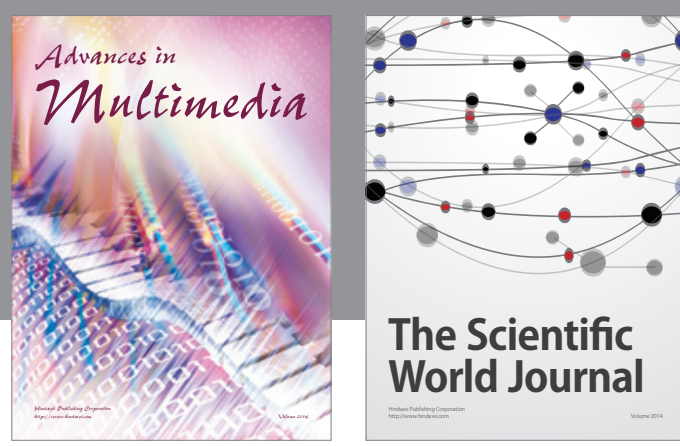

The Scientific World Journal
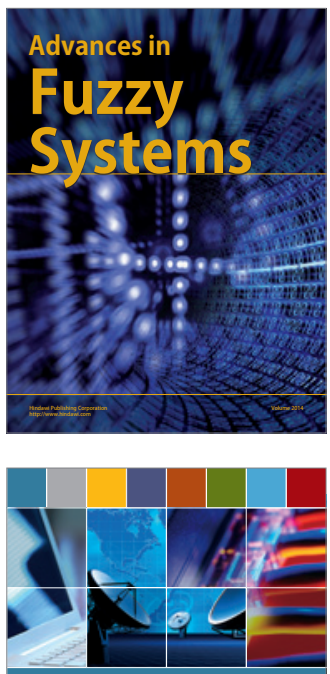

Computer Networks and Communications
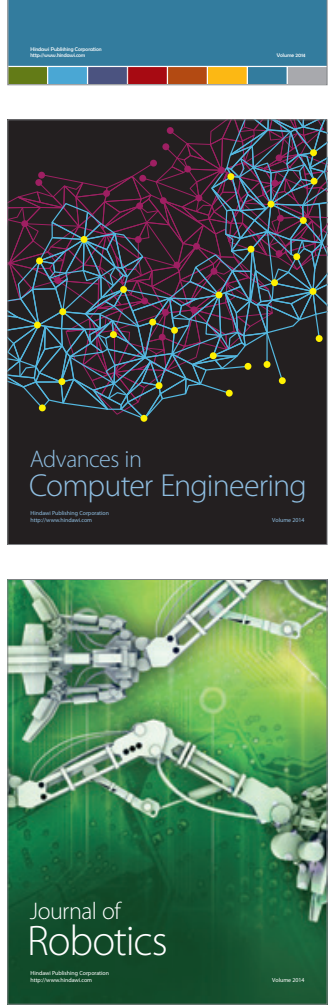
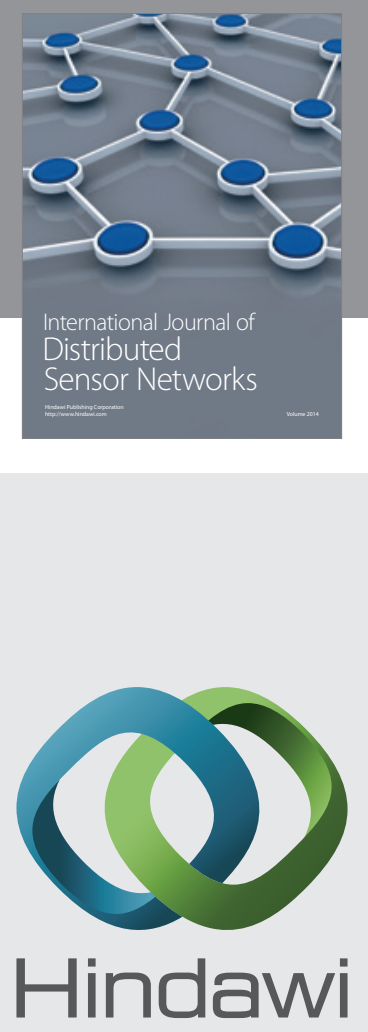

Submit your manuscripts at

http://www.hindawi.com
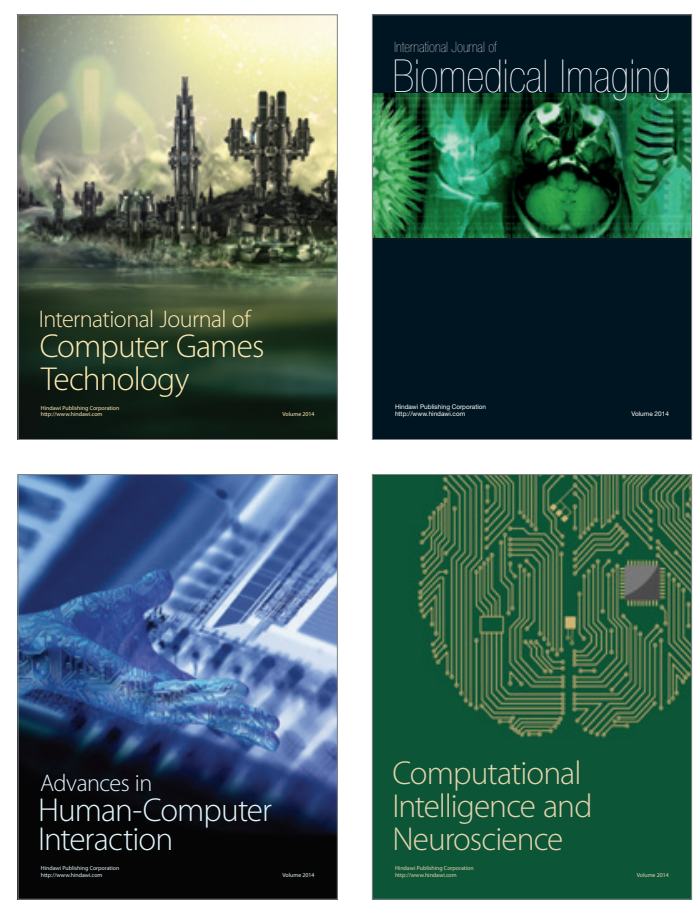
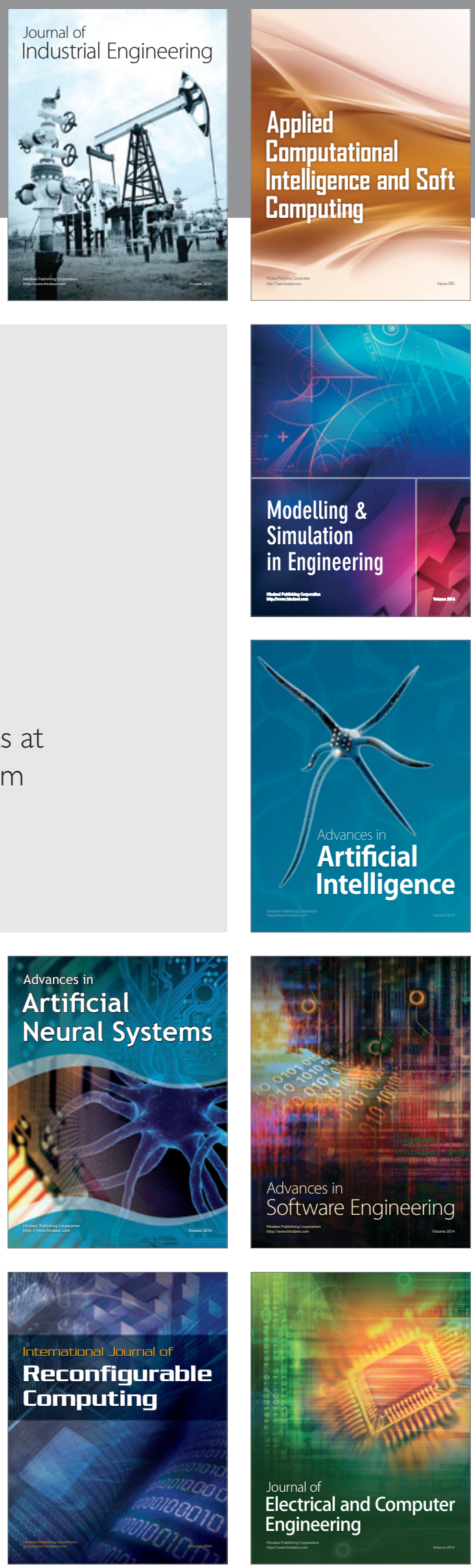